Bio - grafia. Escritos sobre la Biología y su Enseñanza. ISSN 2027

Edición Extraordinaria. p.p. 715 - 728

Memorias del VIII Encuentro Nacional de Experiencias en Enseñanza de la Biología y la Educación Ambiental. III Congreso Nacional de Investigación en Enseñanza de la Biología.

\title{
INFLUENCIA DE LOS ESPACIOS ESCOLARES EN LOS COMPORTAMIENTOS PROAMBIENTALES DE LOS ESTUDIANTES DE TRES COLEGIOS OFICIALES DE BOGOTÁ D.C.
}

\section{INFLUENCE OF SCHOLAR SPACES IN THE STUDENT'S PRO-ENVIRONMENTAL BEHAVIORS IN THREE PUBLIC SCHOOLS OF BOGOTÁ CITY}

Barreto Tovar Carlos Humberto1, Velásquez Olmos Gloria Patricia ${ }^{2}$, Medina Angarita Nancy Fabiola ${ }^{3}$, Moreno Moreno Deisy ${ }^{4}$, Fagua Preciado Ruth Esperanza ${ }^{5}$, Serrato Muñoz Fanny Mireya $^{6}$

\section{Resumen}

En este artículo se presentan aspectos sobre la influencia que tienen los espacios escolares en los comportamientos proambientales de los estudiantes de tres colegios oficiales de la ciudad de Bogotá. Para este trabajo la fase metodológica se desarrolló a través de un enfoque cualitativo, longitudinal y de Investigación Acción Educativa. El problema fue diagnosticado a través de la observación del comportamiento de los estudiantes de los tres colegios, durante un periodo de seis meses. Se utilizaron cinco categorías base para el análisis de las situaciones observadas: conocimientos ambientales, actitudes

\footnotetext{
${ }^{1}$ Universidad de la Sabana. Facultad de Educación. Maestría en Educación. Docente - Investigador. Correo: carloshumbertobarreto@hotmail.com

${ }^{2}$ Universidad de la Sabana. Facultad de Educación. Maestría en Educación. Coinvestigadora. Docente Colegio Gran Yomasa. Sede A. Jornada Mañana. Correo:glopaveol@gmail.com

${ }^{3}$ Universidad de la Sabana. Facultad de Educación. Maestría en Educación. Coinvestigadora. Docente Colegio Gran Yomasa. Sede A. Jornada Mañana. Correo: nancymean@unisabana.edu.co

${ }^{4}$ Universidad de la Sabana. Facultad de Educación. Maestría en Educación. Coinvestigadora. Docente Colegio Gran Yomasa. Sede A. Jornada Mañana. Correo: deissymo@unisabana.edu.co

${ }^{5}$ Universidad de la Sabana. Facultad de Educación. Maestría en Educación. Coinvestigadora. Docente Colegio Gabriel Betancourt Mejía. Sede A. Jornada Tarde. Correo: ruthcita1002@hotmail.com

${ }^{6}$ Universidad de la Sabana. Facultad de Educación. Maestría en Educación. Coinvestigadora. Docente Colegio Manuel Elkin Patarroyo. Sede A. Jornada Mañana. Correo: fannysemu@unisabana.edu.co
} 
Bio - grafia. Escritos sobre la Biología y su Enseñanza. ISSN 2027

Edición Extraordinaria. p.p. 715 - 728

Memorias del VIII Encuentro Nacional de Experiencias en Enseñanza de la Biología y la Educación Ambiental. III Congreso Nacional de Investigación en Enseñanza de la Biología.

proambientales, comportamientos proambientales, cultura ambiental y espacios escolares, además de otras categorías que han sido catalogadas como emergentes durante este proceso de investigación. Se puede concluir que a pesar del conocimiento ambiental que tienen los estudiantes, éste no es suficiente para comportarse proambientalmente en los colegios. Además, se muestra que la planta física tiene influencia en los comportamientos proambientales de los estudiantes, esto evidencia que el espacio escolar juega un papel importante en el comportamiento de los alumnos, puesto que, la relación bidireccional: espacio escolar - comportamiento proambiental, es influenciada y afectada de acuerdo con la percepción positiva o negativa que se tenga de ésta.

Palabras Claves: conocimientos ambientales, actitudes proambientales, comportamientos proambientales, cultura ambiental, espacios escolares.

\begin{abstract}
This article presents some aspects about the influence of scholar spaces in the student's pro-environmental behaviors in three public schools of Bogotá City. For this study the methodological phase was developed through a longitudinal, qualitative perspective and the educational action research. The problem was diagnosed through the observation of the students' behaviors of the three schools, during a six months period. Five base categories were used to analyze the observed situations: environmental knowledge, pro-environmental attitudes, pro-environmental behaviors, environmental culture and scholar spaces. During this research process others categories have been classified like emerging. The results demonstrate even though the students have the environmental knowledge, this is not enough to behave pro-environmentally in the schools. Furthermore it is shown that the infrastructure has influence in the students' pro-environmental behaviors. This indicates that scholar space plays a very important role in the students' behavior, given that the bidirectional relationship: Scholar space - pro-environmental behavior is influenced and affected with the positive or negative perception that anyone has of it.
\end{abstract}

Key words: Environmental knowledge, pro-environmental attitudes, pro-environmental behaviors, environmental culture, scholar spaces. 
Bio - grafia. Escritos sobre la Biología y su Enseñanza. ISSN 2027

Edición Extraordinaria. p.p. 715 - 728

Memorias del VIII Encuentro Nacional de Experiencias en Enseñanza de la Biología y la Educación Ambiental. III Congreso Nacional de Investigación en Enseñanza de la Biología.

\section{Introducción}

Este trabajo presenta elementos correspondientes a los avances de la fase de resultados del proyecto de tesis de Maestría en Educación de la Universidad de la Sabana titulado "Diseño, implementación y evaluación de propuestas de intervención socioeducativa para determinar la influencia de los espacios escolares en los comportamientos proambientales de los estudiantes de tres colegios oficiales de Bogotá D.C."

En las dos últimas décadas la preocupación por la conservación del medio ambiente ha ido en aumento. Muestra de ello fue el encuentro de 189 naciones pertenecientes a la ONU, en la Cumbre del Milenio del año 2000, donde se determinó la importancia de generar acciones encaminadas al desarrollo sostenible debido a los evidentes problemas de deterioro ambiental. La educación tiene responsabilidad legal para contribuir a la resolución de específicos problemas ambientales (Obregoso y Vallejo, 2010) y aunque ésta direcciona sus esfuerzos hacia la formación de los estudiantes en el conocimiento y actitudes frente al medio ambiente, no se ve reflejado en sus comportamientos, ya que, "Los problemas ambientales poseen causas conductuales" (Corral, 2006, p.113).

Las categorías definidas para la investigación son: cultura ambiental, conocimiento ambiental, actitudes ambientales, comportamientos proambientales y espacio escolar.

\begin{tabular}{|c|c|c|}
\hline CATEGORÍA & OBJETIVO & DEFINICIÓN \\
\hline $\begin{array}{l}\text { Actitudes } \\
\text { ambientales }\end{array}$ & $\begin{array}{l}\text { Conocer algunas actitudes } \\
\text { ambientales que los } \\
\text { estudiantes tienen en su } \\
\text { relación con la institución } \\
\text { escolar }\end{array}$ & $\begin{array}{l}\text { Predisposición del pensamiento humano a actuar } \\
\text { en favor o en contra del entorno social, } \\
\text { teniendo como base las vivencias, los } \\
\text { conocimientos y los valores del individuo con } \\
\text { respecto a su entorno. Febles, M. (2001). }\end{array}$ \\
\hline $\begin{array}{l}\text { Conocimientos } \\
\text { ambientales }\end{array}$ & $\begin{array}{l}\text { Identificar los conocimientos } \\
\text { que tienen los estudiantes } \\
\text { sobre el cuidado del medio }\end{array}$ & $\begin{array}{l}\text { Proceso complejo que incluye la obtención, } \\
\text { análisis y sistematización, por parte del } \\
\text { individuo, de la información proveniente de su }\end{array}$ \\
\hline
\end{tabular}


Bio - grafia. Escritos sobre la Biología y su Enseñanza. ISSN 2027

Edición Extraordinaria. p.p. 715 - 728

Memorias del VIII Encuentro Nacional de Experiencias en Enseñanza de la Biología y la Educación Ambiental. III Congreso Nacional de Investigación en Enseñanza de la Biología.

\begin{tabular}{|l|l|l|}
\hline & ambiente. & entorno. Febles, M. (2001). \\
\hline $\begin{array}{l}\text { Comportamientos } \\
\text { proambientales }\end{array}$ & $\begin{array}{l}\text { Identificar los } \\
\text { comportamientos } \\
\text { proambientales que los } \\
\text { estudiantes presentan en } \\
\text { algunas situaciones de su vida } \\
\text { cotidiana. }\end{array}$ & $\begin{array}{l}\text { Conjunto de acciones deliberadas y efectivas } \\
\text { que responden a requerimientos sociales e } \\
\text { individuales cuya consecuencia es la protección } \\
\text { del medio ambiente. Corral, V. (2001). }\end{array}$ \\
\hline $\begin{array}{l}\text { Cultura } \\
\text { ambiental }\end{array}$ & $\begin{array}{l}\text { Determinar el nivel de cultura } \\
\text { ambiental que poseen los } \\
\text { estudiantes. }\end{array}$ & $\begin{array}{l}\text { Conjunto de formas de comunicación del hombre } \\
\text { y el grupo social con el universo. Implica la } \\
\text { creación de nuevos lenguajes que le permiten al } \\
\text { hombre interactuar con su entorno, fusionarse } \\
\text { con la naturaleza y asumir una posición de } \\
\text { protección del medio ambiente. Rodríguez, J. } \\
\text { (2004). }\end{array}$ \\
\hline Espacio Escolar & $\begin{array}{l}\text { Reconocer la relación que } \\
\text { tienen los estudiantes con su } \\
\text { espacio escolar }\end{array}$ & $\begin{array}{l}\text { Relación existente entre arquitectura escolar e } \\
\text { innovación pedagógica. Define el espacio escolar } \\
\text { como agente educativo que posibilita ambientes } \\
\text { de aprendizaje y bienestar para la educación } \\
\text { del hombre completo. Salmerón, H. (1992). } \\
\text { Ruiz, J.M. (1994). }\end{array}$ \\
\hline
\end{tabular}

Fuente: Equipo investigador.

Los espacios físicos, según Santos (1977), se cargan de significado a través de los usos que la cultura les atribuye, y por esta razón, se debería investigar sobre el valor del espacio como factor educativo, ya que, es necesario hacer de las escuelas lugares acogedores para el profesorado y para el alumnado, con el fin de que dichos lugares, sean considerados propios, porque la participación alcanza a su uso democrático, a su adorno peculiar, a su exquisita limpieza y a su vivencia compartida. Por otra parte, la arquitectura escolar, "facilita 0 inhibe el desarrollo de determinadas funciones, movimientos, relaciones, ejecución de tareas en suma, es ese ámbito físico y Social en donde se transmiten valores, promueven identidades personales y grupales, y se favorecen formas de hacer $y$ de relacionarse" (Salmerón, 1992). En este orden de ideas, creemos que los espacios físicos 
Bio - grafia. Escritos sobre la Biología y su Enseñanza. ISSN 2027

Edición Extraordinaria. p.p. 715 - 728

Memorias del VIII Encuentro Nacional de Experiencias en Enseñanza de la Biología y la Educación Ambiental. III Congreso Nacional de Investigación en Enseñanza de la Biología.

ejercen una influencia en los comportamientos de los estudiantes, específicamente, en los alumnos de tres colegios públicos de la ciudad de Bogotá, que puede ser de carácter positivo: proambiental o negativo: no ambiental, evidenciados en el quehacer diario en dichas instituciones. Si se consigue modificar algunos comportamientos de los estudiantes durante el proceso de cambio y transformación de comportamientos no ambientales por comportamientos proambientales, se logrará una influencia bidireccional en los distintos espacios escolares que ofrece las instituciones, enriqueciendo la cultura ambiental.

Si se consigue modificar algunos comportamientos de los estudiantes durante el proceso de cambio y transformación de comportamientos no ambientales por comportamientos proambientales, se logrará una influencia bidireccional en los distintos espacios escolares que ofrece las instituciones, enriqueciendo la cultura ambiental.

En esa línea, este trabajo está encaminado a identificar la influencia de los espacios escolares en los comportamientos proambientales de los estudiantes de los colegios oficiales de Bogotá: Gran Yomasa, Gabriel Betancourt Mejía y Manuel Elkin Patarroyo.

\section{METODOLOGÍA}

Para este trabajo la fase metodológica se desarrolló a través de un enfoque cualitativo, longitudinal y de Investigación Acción Educativa. Para Lewin (1946) la Investigación Acción, está dirigida a la dirección y construcción de la sociedad, permitiendo la comparación de los efectos de varias formas de acción social, pues, es posible modificar ciertas conductas sociales de los grupos. Es así, como la participación es el único modo para lograr el poder de control sobre el grupo, que a su vez, favorece las relaciones interpersonales y el compromiso individual, garantizando la obtención de los resultados previstos para la práctica. El papel del investigador será participante, haciendo una exploración permanente de distintos fenómenos, en especial el relacionado con los comportamientos no ambientales y proambientales que presentan los estudiantes, con el fin de elaborar el diagnóstico que permita la planificación, organización y ejecución de una propuesta pedagógica de 
Bio - grafia. Escritos sobre la Biología y su Enseñanza. ISSN 2027

Edición Extraordinaria. p.p. 715 - 728

Memorias del VIII Encuentro Nacional de Experiencias en Enseñanza de la Biología y la Educación Ambiental. III Congreso Nacional de Investigación en Enseñanza de la Biología.

intervención, encaminada a la transformación de esta realidad social que se evidencia en la escuela.

Este trabajo se desarrolló en los siguientes colegios:

\section{Colegio Gran Yomasa IED}

Es una institución ubicada en el barrio Gran Yomasa de la localidad $5^{a}$ de Usme. Se ubica en la Calle $81 C$ sur No 2 bis 00 Este. Sede única. Modalidad académica.

\section{Colegio Gabriel Betancourt Mejía}

Institución ubicada en el la carrera $87^{\circ}$ A N $N^{\circ} 6$ A-23 en la localidad $8^{\circ}$, Kennedy en el barrio El Tintal. Cuenta actualmente con dos sedes A y B. Tiene dos jornadas en cada una, mañana y tarde con modalidad académica desde primera infancia hasta grado once.

\section{Colegio Manuel Elkin Patarroyo}

Es una institución ubicada en el barrio La Perseverancia de la localidad $3^{a}$ de Santafé, UPZ Macarena, en la Carrera $4 N^{\circ} 32$ - 90. Sede única.

Para este trabajo, en las tres instituciones educativas: Gran Yomasa, Gabriel Betancourt Mejía y Manuel Elkin Patarroyo, se utilizó la observación como método de recolección de la información, que "implica adentrarnos en profundidad a situaciones sociales y mantener un papel activo, así como una reflexión permanente. Estar atento a los detalles, sucesos, eventos e interacciones" (Hernández, Fernández \& Baptista, 2006), observaciones que quedaron registradas en los diarios de campo, los cuales según Rodríguez, Gil \& García (1999), son apuntes para recordar la observación realizada, con el fin de facilitar un posterior estudio y reflexión sobre el problema.

Los registros se realizaron desde septiembre de 2014 hasta abril de 2015, durante este tiempo, se observaron las actitudes y conductas de los estudiantes de los tres Colegios, con respecto a su relación con el entorno, el espacio escolar y el ambiente en general. La observación se realizó durante la jornada escolar, en cualquier momento, en distintos lugares de los colegios: salones de clases, baños, patios, zonas verdes, espacios comunes, 
Bio - grafia. Escritos sobre la Biología y su Enseñanza. ISSN 2027

Edición Extraordinaria. p.p. 715 - 728

Memorias del VIII Encuentro Nacional de Experiencias en Enseñanza de la Biología y la Educación Ambiental. III Congreso Nacional de Investigación en Enseñanza de la Biología.

además, fue intencional y por conveniencia. No obstante, aunque la observación se hizo durante el día, la hora de descanso y la hora de toma de refrigerio de los estudiantes, fue el espacio de mayor observación, puesto que, la interacción de los estudiantes entre sí, permitió obtener diversas apreciaciones de las actitudes y comportamientos de los niños y niñas.

Cada observación se registró en un diario de campo, 117 en total, donde las docentes investigadoras describían las situaciones observadas, hacían su propia interpretación, algunas con registro fotográfico y audiovisual, y categorizaban los registros de acuerdo con las categorías planteadas para el problema: conocimientos ambientales, actitudes proambientales, comportamientos proambientales, cultura ambiental, espacios escolares, permitiendo la clasificación de distintas categorías emergentes durante el análisis de las diversas situaciones. Este proceso de observación y registro de datos en el diario de campo, permitió el diagnóstico del problema de investigación, ofreciendo información sobre la influencia de los espacios escolares en el comportamiento proambiental de los estudiantes de los tres colegios distritales y su relación bidireccional. Por otra parte, se realizó un proceso de recolección de información a partir de una entrevista realizada a algunos integrantes del Comité Ambiental Escolar de cada institución.

\section{RESULTADOS Y DISCUSIÓN}

Al realizar el análisis de las actitudes y conductas de los estudiantes de tres colegios públicos de la ciudad de Bogotá: Gran Yomasa, Manuel Elkin Patarroyo y Gabriel Betancourt Mejía, registrados en 117 diarios de campo realizados desde septiembre de 2014 hasta abril de 2015, con respecto a su relación con el entorno, el espacio escolar y el ambiente en general, se reconocen varios hallazgos importantes para el proceso de investigación. Es necesario aclarar que los tres colegios seleccionados presentan diferencias en cuanto a la antigüedad de la infraestructura de la institución. El Colegio Gran Yomasa ha pasado por un proceso de sustitución de la planta física, el Colegio Manuel Elkin Patarroyo es el más antiguo (1936) y el Colegio Gabriel Betancourt Mejía, posee una estructura de aproximadamente diez años. 
Bio - grafia. Escritos sobre la Biología y su Enseñanza. ISSN 2027

Edición Extraordinaria. p.p. 715 - 728

Memorias del VIII Encuentro Nacional de Experiencias en Enseñanza de la Biología y la Educación Ambiental. III Congreso Nacional de Investigación en Enseñanza de la Biología.

Dentro de los hallazgos realizados se observa que, aunque las categorías base determinadas para el problema: Cultura ambiental, conocimiento ambiental, Actitud ambiental, comportamientos proambientales y Planta física, enmarcaron la mayor parte de las observaciones, éstas no fueron suficientes para el análisis y reflexión del problema, ya que en los registros emergieron otras categorías diferentes, que apoyan el planteamiento del problema sobre la influencia de los espacios escolares en los comportamientos proambientales de los estudiantes.

En la tabla 1, se observa la estadística consolidada de los tres colegios, donde se evidencia la frecuencia de las categorías mencionadas, las cuales son la base para el análisis del problema. Inicialmente se planteó el estudio de las observaciones y diarios de campo desde las categorías determinadas; sin embargo, se comprueba que los aspectos recurrentes corresponden al lado negativo de algunas categorías. Es así, como los registros realizados denotan mayor frecuencia de las actitudes no ambientales que las actitudes ambientales positivas; de la misma manera, la falta de cultura ambiental es más evidente en las observaciones, que la cultura ambiental, así mismo, los comportamientos proambientales son menores que los no ambientales. No obstante, en los registros realizados se observa que el conocimiento ambiental es más frecuente que la falta de conocimiento ambiental. Además, se evidencian categorías emergentes como: contaminación auditiva y del aire y cuidado de la vida. 
Bio - grafia. Escritos sobre la Biología y su Enseñanza. ISSN 2027

Edición Extraordinaria. p.p. 715 - 728

Memorias del VIII Encuentro Nacional de Experiencias en Enseñanza de la Biología y la Educación Ambiental. III Congreso Nacional de Investigación en Enseñanza de la Biología.

\begin{tabular}{|c|c|c|}
\hline CATEGORÍAS & FRECUENCIA & PORCENTAJE $\%$ \\
\hline Cultura ambiental & 15 & 5,54 \\
\hline Conocimiento ambiental & 27 & 9,96 \\
\hline Actitudes ambientales positivas & 15 & 5,54 \\
\hline Comportamientos Proambientales & 32 & 11,81 \\
\hline Planta física & 67 & 24,72 \\
\hline Comportamientos No ambientales & 48 & 17,71 \\
\hline Falta de conocimiento ambiental & 10 & 3,69 \\
\hline Contaminación (auditiva y del aire) & 5 & 1,85 \\
\hline Falta de cultura ambiental & 22 & 8,12 \\
\hline Actitud no ambiental & 27 & 9,96 \\
\hline Cuidado de la vida & 3 & 1,11 \\
\hline
\end{tabular}

Tabla 1. Categorías arrojadas por los diarios de campo.

En la gráfica 1 se observan las categorías arrojadas por los registros de los diarios de campo, así mismo, en la gráfica 2, se evidencian estos datos, de acuerdo con el porcentaje que le corresponde a cada categoría base y emergente

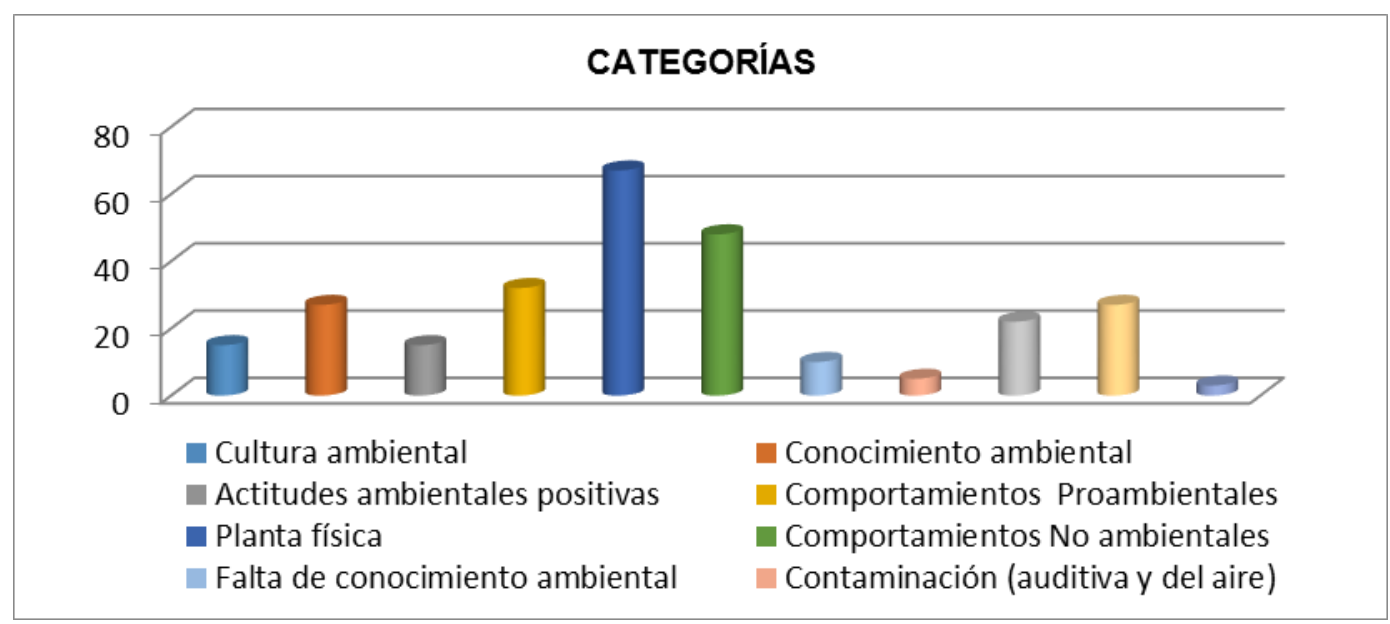


Bio - grafia. Escritos sobre la Biología y su Enseñanza. ISSN 2027

Edición Extraordinaria. p.p. 715 - 728

Memorias del VIII Encuentro Nacional de Experiencias en Enseñanza de la Biología y la Educación Ambiental. III Congreso Nacional de Investigación en Enseñanza de la Biología.

Gráfica 1. Categorías arrojadas por los diarios de campo, subdivididas positiva y negativamente, de acuerdo con el número de registros realizados en los diarios de campo.

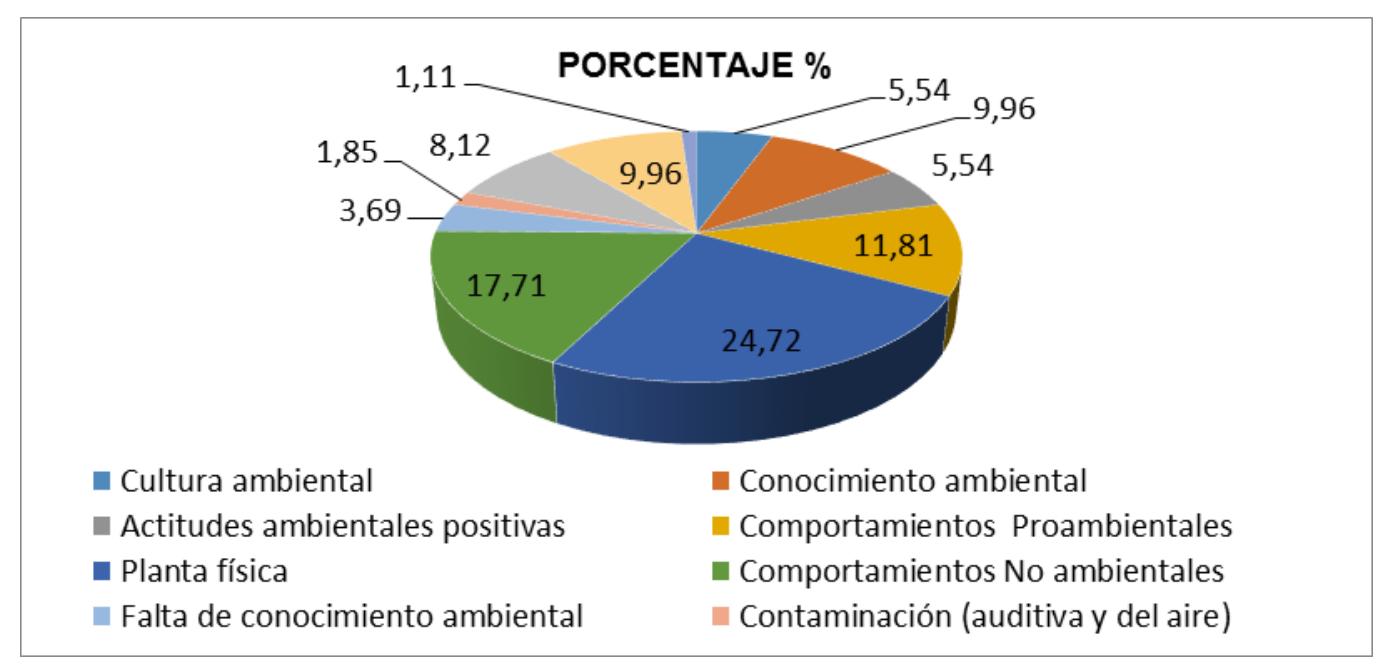

Gráfica 2. Porcentajes equivalentes a las observaciones y registros realizados en los diarios de campo por categorías.

Teniendo en cuenta la tabla 1 con sus respectivas representaciones, se puede determinar que: la cultura ambiental, una de las categorías predeterminadas para esta investigación, se evidencia en un 5,54\%, de las observaciones, contrario a esto, la falta de cultura ambiental es más evidente en las situaciones observadas; ya que, el 8,12\% de los registros arrojó comportamientos donde falta cultura ambiental. Issac- Márquez \& Salivaría (2011) definen cultura ambiental como el conjunto de actitudes, intenciones de comportamiento y conocimientos ambientales.

Por otra parte, Rodríguez (2004) afirma que la cultura ambiental, implica la creación de nuevos lenguajes que le permiten al hombre interactuar con su entorno, fusionarse con la naturaleza y asumir una posición de protección del medio ambiente. Teniendo en cuenta estas definiciones, podemos afirmar que la cultura ambiental es una categoría muy amplia, en ocasiones difícil de evidenciar en los estudiantes, ya que, reúne a otras categorías y generalmente, esta cultura es fortalecida por los padres y su formación en la casa. No 
Bio - grafia. Escritos sobre la Biología y su Enseñanza. ISSN 2027

Edición Extraordinaria. p.p. 715 - 728

Memorias del VIII Encuentro Nacional de Experiencias en Enseñanza de la Biología y la Educación Ambiental. III Congreso Nacional de Investigación en Enseñanza de la Biología.

obstante, de los 117 diarios de campo que realizamos, se pudo evidenciar la cultura ambiental en 15 momentos.

Un ejemplo claro es el registrado en un diario de campo del Colegio Manuel Elkin Patarroyo "Llega una señora de servicios generales a coordinación. Me dice que si puedo acompañarla que necesita mostrarme algo. Voy detrás de ella, ingresa al baño de los niños del segundo piso y me señala el orinal de los niños. Al observar miro en uno de los orinales un vaso de helado que les habían dado en el refrigerio lleno de orines y en el otro orinal había una cáscara de granadilla"

Los comportamientos proambientales son determinados como otra de las categorías base para el análisis del problema de investigación. Según Stern (2000), "el comportamiento ambiental de los individuos se da a través de variables causales, los valores de una persona es la variable más importante para definir un comportamiento ambiental que le permiten llevar a cabo acciones en beneficio del ambiente". En los registros se evidencia que solamente un $11,81 \%$ de los comportamientos observados son proambientales, mientras que el $17,71 \%$ de los comportamientos registrados son No ambientales. Uno de los representantes del Comité Ambiental Escolar del grado séptimo del Colegio Gran Yomasa, al referirse a los comportamientos de los estudiantes afirma: "Ven una mesa limpia, bien bonita y de todas maneras la rayan, botan basura, los muchachos juegan con las canecas. Muchos ponen las manos en las llaves y ya las quitan, de todas maneras, el agua se sigue botando."

En cuanto a las actitudes ambientales observadas en los estudiantes, solamente se registran $5,54 \%$ de las observaciones y un $9,96 \%$ en las actitudes no ambientales. Para Febles (1999) las actitudes ambientales, deben definirse como una "predisposición del pensamiento humano a actuar a favor o en contra del entorno social, teniendo como base las vivencias, los conocimientos y los valores del individuo con respecto a su entorno; estas no solo se proyectan en una dirección determinada, también poseen un nivel de intensidad fuerte o débil"; es así, como las actitudes negativas o no ambientales se hacen más evidentes en la relación de los estudiantes con el medio que los rodea, específicamente con los espacios escolares de los tres colegios. Una de las integrantes del Comité ambiental 
Bio - grafia. Escritos sobre la Biología y su Enseñanza. ISSN 2027

Edición Extraordinaria. p.p. 715 - 728

Memorias del VIII Encuentro Nacional de Experiencias en Enseñanza de la Biología y la Educación Ambiental. III Congreso Nacional de Investigación en Enseñanza de la Biología.

escolar afirma: "Muchos no saben tratar con la naturaleza. Hay artos que tratan de tener este colegio limpio, pero hay otros que no le ponen interés", al entrevistarla y preguntarle sobre las actitudes de los compañeros en el Colegio Gran Yomasa.

Por otro lado, se evidencia conocimiento ambiental por parte de los estudiantes en un $9,96 \%$ de los registros, sin embargo, el 3,69\% de las observaciones, arrojan que la falta de conocimiento ambiental conlleva a ciertas actuaciones de los niños. Para Febles (1999) el conocimiento ambiental es "un proceso complejo, que incluye la obtención, análisis y sistematización por parte del individuo de la información proveniente de su entorno, social por naturaleza, este constituye un paso importante para su comprensión a través de acciones concretas, que a su vez, influyen en el desarrollo de estos conocimientos".

En las instituciones escolares se brindan y afianzan conocimientos ambientales, un ejemplo de esto, es el registrado en un diario de campo en el Colegio Gabriel Betancurt Mejía: "Siendo las 3: 30 de la tarde y por sugerencia de los niños empezamos a elaborar nuevamente la cartelera de aire puro. Les hice un recuento de los beneficios del aire, les vivencié la correcta respiración, respiración en cuatro tiempos y cantaron la canción que creé para este tópico. Cada estudiante recibió una hoja de papel blanco, lápiz, colores, marcadores y elaboraron dibujos alusivos al aire puro, algunos con frases sencillas. Se pegaron sobre papel periódico y con letreros adicionales en marcador elaborados por la niña de servicio social: Nicolle y otros por mí".

La planta física y los espacios escolares son una categoría que se hace reiterativa en un $24,72 \%$ de las observaciones, siendo el porcentaje más alto de los registros; ya que, ésta cobra importancia en su relación bidireccional con los estudiantes, pues, el espacio escolar, según Salmerón (1999) actúa como agente educativo que posibilita ambientes de aprendizaje y bienestar para la educación del hombre completo. "Los niños de primaria tratan de comprar a la hora de descanso, el lugar destinado para mostrador de los productos está a 1 metro 30 centímetros y los niños pequeños no alcanzan, por esta razón, deben buscar mecanismos para ayudarse entre ellos y así alcanzar y poder ser atendidos. Una de las estrategias es colgarse de la baranda y la otra estrategia muy común, es alzarse 
Bio - grafia. Escritos sobre la Biología y su Enseñanza. ISSN 2027

Edición Extraordinaria. p.p. 715 - 728

Memorias del VIII Encuentro Nacional de Experiencias en Enseñanza de la Biología y la Educación Ambiental. III Congreso Nacional de Investigación en Enseñanza de la Biología.

entre compañeros y sostenerse mientras compra, uno a uno". Tomado del diario de campo del Colegio Gran Yomasa.

Algunas categorías emergieron en las observaciones realizadas, tales como: cuidado de la vida con un $1,11 \%$ de los registros y contaminación tanto auditiva, como del aire con un $1,85 \%$ (siendo evidenciadas estas últimas en el Colegio Gran Yomasa, especialmente, en el año 2014, mientras que se construía la nueva planta física del colegio).

\section{CONCLUSIONES}

El análisis de las observaciones realizadas evidencia la recurrencia de los estudiantes en los aspectos negativos de algunas categorías; es así, como se observan más acciones que denotan falta de cultura ambiental, actitudes y comportamientos no ambientales.

A pesar del conocimiento ambiental que tienen los estudiantes, éste no es suficiente para comportarse proambientalmente en los colegios, ya que, el porcentaje de actitudes y comportamientos no ambientales es mayor que el número de actitudes y comportamientos proambientales. Es decir, no es garantía que una persona se comporte proambientalmente por el nivel de conocimientos ambientales que posea.

La planta física tiene influencia en los comportamientos proambientales de los estudiantes, esto evidencia que el espacio escolar juega un papel importante en el comportamiento de los alumnos, puesto que, la relación bidireccional: espacio escolar - comportamiento proambiental, es influenciada y afectada de acuerdo con la percepción positiva o negativa que se tenga de ésta.

\section{REFERENCIAS}

- Corral, V. (2001). Comportamiento ambiental. Una introducción al estudio de las conductas protectoras del ambiente. Santa Cruz de Tenerife España: RESMA 
Bio - grafia. Escritos sobre la Biología y su Enseñanza. ISSN 2027

Edición Extraordinaria. p.p. 715 - 728

Memorias del VIII Encuentro Nacional de Experiencias en Enseñanza de la Biología y la Educación Ambiental. III Congreso Nacional de Investigación en Enseñanza de la Biología.

- Corral, V. (2006). Contribuciones del Análisis de la Conducta a la Investigación del Comportamiento Pro-Ecológico. Revista Mexicana del Análisis de la Conducta,32, 11127.

- Febles, M. (2001). Hacia un enfoque holístico del medio ambiente desde la psicología ambiental. Facultad de Psicología: Universidad de la Habana.

- Hernández, R., Fernández, C. \& Baptista, P. Metodología de la Investigación. México: Mc Graw Hill. Quinta Edición.

- Lewin, K. (1946).Action research and minority problems. Journal for Social Issues, 2(4), 34-46.

- Isaac-Márquez, A., \& Manzanero Acevedo, L. (2011). Cultura ambiental en estudiantes de bachillerato. Estudio de caso de la educación ambiental en el nivel medio superior de Campeche. (Spanish). Revista Electrónica De Investigación Educativa, 13(2), 83-99.

- Obregoso, A. y Vallejo, Y. (2010). Dos décadas de la Educación Ambiental en Colombia (1990-2010). Una mirada desde la normatividad. Colombia. Revista Actualidades Investigativas en Educación, 81-95.

- Rodríguez. G., Gil. J. \& García. E. (1999). Metodología de la Investigación Cualitativa. Málaga. Universidad del Pacífico.

- Rodríguez, J. (2004). Anuario de ecología, cultura y sociedad. Año 1, Núm. 1, 2001.

- Ruiz, J.M. (1994). El espacio escolar. Revista Complutense de Educación. Vol. 5. Editorial Complutense. Madrid.

- Salmerón, H. (1992). Evaluación de los espacios arquitectónicos escolares. Granada, España: Universidad de Granada.

- Santos, M.Á. (1977). El espacio como factor educativo. Revista Española de Pedagogía, 135.

- Stern (2000). Toward a Coherent Theory of Environmentally Significant Behavior. Master Thesis. 